\title{
Low-Profile Circularly Polarized Antenna Exploiting Fabry-Perot Resonator Principle
}

\author{
Kamil PITRA, Zbynek RAIDA, Jaroslav LACIK \\ Dept. of Radio Electronics, Brno University of Technology, Technicka 12, 61600 Brno, Czech Republic \\ raida@feec.vutbr.cz
}

\begin{abstract}
We designed a patch antenna surrounded by a mushroom-like electromagnetic band-gap (EBG) structure and completed it by a partially reflective surface (PRS). EBG suppresses surface waves and creates the bottom wall of the Fabry-Perot (FP) resonator. PRS plays the role of a planar lens and forms the top wall of the FP resonator. The novel PRS consists of a two-layer grid exhibiting inductive and capacitive (LC) behavior which allows us to obtain a reflection phase between $-180^{\circ}$ and $+180^{\circ}$. Thanks to this PRS, we can control the height of the cavity in the range from $\lambda / 2$ to $\lambda / 300$.
\end{abstract}

Obtained results show that the FP resonator antenna enables us to achieve a low profile and a high-gain. The patch is excited by a microstrip transmission line via the crossslot aperture generating the circular polarization.

Functionality of the described concept of the FP antenna was verified at $10 \mathrm{GHz}$. The antenna gain was $15 \mathrm{dBi}$, the impedance bandwidth $2.3 \%$ for $\left|S_{11}\right|<-10 \mathrm{~dB}$, and the axial ratio bandwidth $0.6 \%$ for $A R<3.0 \mathrm{~dB}$. Hence, the antenna is suitable for narrowband applications.

Computer simulations show that the microwave FP antenna can be simply redesigned to serve as a source of circularly polarized terahertz waves.

\section{Keywords}

High gain antenna, Fabry-Perot resonator, terahertz source, partially reflective surface, mushroom-like electromagnetic band-gap structure, circular polarization

\section{Introduction}

The gain of an antenna can be increased by a partially reflective superstrate (PRS) [1-4] which behaves like a planar lens. If the planar antenna is surrounded by an electromagnetic band-gap (EBG) structure to suppress surface waves, the PRS and the EBG can form a cavity behaving like a Fabry-Perot (FP) resonator.

A ray method was applied to demonstrate the function of the FP antenna [1]. Both the EBG and the PRS are described by complex reflection coefficients $\exp \left(j \varphi_{1}\right)$ and $\rho \cdot \exp \left(j \varphi_{2}\right)$, respectively. Both the surfaces can be assumed to be homogeneous, and edge effects can be neglected.

Many researchers assume infinitely large ground plane and PRS for such an analysis. Obviously, this assumption is practically valid for very large PRS and ground plane areas, which are in order of $25 \lambda^{2}$ to $36 \lambda^{2}$. In a fact, small areas have found to be useful to increase the bandwidth due to the radiation from the edges [16].

According to the ray method, the resonance condition can be expressed:

$$
h=\frac{\varphi_{1}+\varphi_{2}}{180^{\circ}} \cdot \frac{\lambda}{4}+N \cdot \frac{\lambda}{2} \quad N=0,1,2, \ldots
$$

Here, $\lambda$ is operating wavelength in free space, $\varphi_{1}$ denotes reflection phase of the EBG in degrees, and $\varphi_{2}$ is reflection phase of the PRS in degrees. According to (1), the maximum power $S_{\max }$ in the forward direction is

$$
S_{\max }=\frac{1+\rho}{1-\rho} S_{0} .
$$

Here, $S_{0}$ corresponds to the forward power propagating from the source without the PRS. Then, the height of the resonant cavity $h$ is determined by the reflection phase of the $\operatorname{EBG} \varphi_{1}$, the reflection phase of the PRS $\varphi_{2}$ and the operating wavelength $\lambda$. If (1) is met, the higher the reflection coefficient $\rho$ is, the higher is the forward power $S_{\max }$.

For a conventional FP resonator antenna with a perfectly conductive ground, the condition $\varphi_{1}=\varphi_{2}=180^{\circ}$ is applicable and the distance between the EBG and the PRS is strictly $\lambda / 2$ at the operating frequency. If the cavity height is required to be decreased, the reflection phase of the EBG or the PRS has to be changed.

In [5] and [6], authors exploited an artificial magnetic conductor to surround the antenna. Hence, $\varphi_{1} \approx 0^{\circ}$, and the FP antenna can be created by the cavity which is $\lambda / 4$ high. Using a PRS as a cover, $\varphi_{2} \approx 0^{\circ}$, and the height of the cavity can be reduced to $\lambda / 30$ [7], [8].

Circularly polarized antennas with a low profile are highly demanded. In order to generate circularly polarized waves, two types of feeding techniques are commonly used - a single-feed type and a dual-feed type. In case of the single-feed technique, an external $90^{\circ}$ phase shifter (feed network) is not required. 
In our design, we exploit crossed slots with different lengths for the excitation of a square patch to generate the circular polarization. That way, two near-degenerate orthogonal modes $\left(\mathrm{TM}_{10}\right.$ and $\left.\mathrm{TM}_{01}\right)$ can be achieved. Obviously, the resonant frequency of the patch depends on the length of coupling slots [9], [10]. Both the lengths of crossed slots and their proper ratio have to be carefully adjusted to excite the fundamental resonance of the square patch and to reach required polarization at the same time.

Researching the open literature, we can find the following concepts of circularly polarized Fabry-Perot antennas which have been published recently:

- In [11], authors presented a single-layer partially reflecting surface antenna with PRS cells consisting of rectangular patches. The FP resonance condition was satisfied at the operating frequency approximately. The PRS was used as the antenna superstrate to achieve high reflectivity. Attention was turned to the conversion of the linearly polarized excitation wave to highly directive circularly-polarized radiation over a narrow frequency band. The antenna height was $\lambda / 2$.

- In [12], the bottom wall of the FP resonator was created by the ground plane of a reconfigurable patch antenna providing the right-hand polarization or the left-hand one. The top wall of the FP resonator was formed by a planar lens consisting of non-equidistant patches of different dimensions. The height of the antenna was $\lambda / 2$.

- In [13], authors described a full integration of the circularly polarized FP antenna into the substrate. The ground plane of the patch antenna played the role of the bottom wall of the FP resonator. A double-layered metamaterial structure played the role of the PRS. That way, the height of the antenna was reduced to $\lambda / 3$. The gain reached $15 \mathrm{dBi}$ considering both dielectric and resistive losses.

- In [14], a patch of shorting pins acted as an antenna. PRS was tuned to produce the maximum gain of the radiator. The height of the antenna was $\lambda / 2$. The gain reached $17 \mathrm{dBi}$ considering both dielectric and resistive losses.

In this paper, we describe a novel design of a circularly polarized FP resonator antenna with a two-layer LC superstrate and a mushroom-like EBG structure surrounding the patch. Novel, original approaches used for the design of the presented antenna comprise:

- An exploitation of a mushroom-like EBG structure surrounding the patch for the suppression of surface waves on one hand, and for forming the bottom plane of the FP resonator on the other hand. Suppression of surface waves can increase the gain of the antenna.

- An exploitation of a novel LC superstrate allowing us to obtain an arbitrary phase shift of the reflection co- efficient between $+180^{\circ}$ and $-180^{\circ}$. Changing the phase shift of the reflection coefficient, the height of the FP resonator can be arbitrarily varied. Therefore, the height of the antenna can be significantly reduced.

The described antenna was simulated and measured at the operating frequency $f=10 \mathrm{GHz}$. In the second step, the microwave antenna was shown to be simply redesigned as a source of circularly polarized terahertz wave. Computer simulations proved the developed concept of the terahertz radiator.

\section{Structure of Developed Antenna}

The investigated antenna consists of the partially reflective superstrate (PRS), the mushroom-like electromagnetic bandgap (EBG) structure surrounding the square patch, and the patch. The patch is excited by the microstrip transmission line on the bottom side of the structure via the crossed-slot aperture (see Fig. 1).

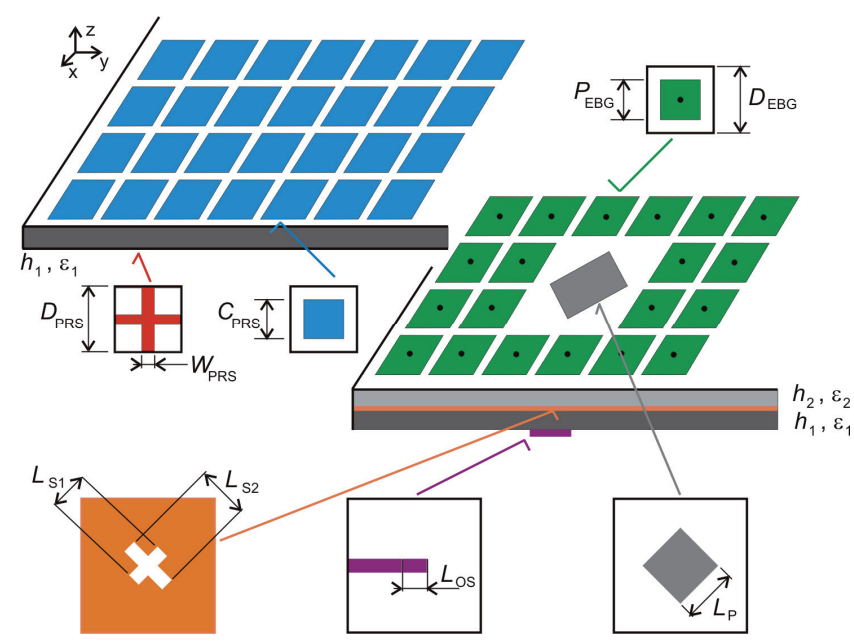

Fig. 1. Structure of the antenna. The patch is excited by the microstrip transmission line (magenta) via crossed slots in the ground plane (orange; the bottom substrate). The patch (gray) is surrounded by the mushroom-like EBG (green; the middle substrate) and covered by the LC PRS (blue capacitors on the top side and red inductors on the bottom side of the top substrate).

The side of the square patch is $L_{P}=6.17 \mathrm{~mm}$ and metallization thickness is $t=0.035 \mathrm{~mm}$. The patch is designed for a substrate with the height $h_{2}=0.762 \mathrm{~mm}$ and the relative permittivity $\varepsilon_{r 2}=3.38$. The microstip feeding line of the impedance $Z_{0}=50 \Omega$ and the matching line, which is $L_{O S}=1.400 \mathrm{~mm}$ long, are etched on a substrate with the height $h_{1}=1.016 \mathrm{~mm}$ and the relative permittivity $\varepsilon_{r 1}=10.2$. The patch is excited via the cross-slot aperture. Lengths of the cross-slot arms are $L_{S 1}=4.060 \mathrm{~mm}$ and $L_{S 2}=3.660 \mathrm{~mm}$. The center of the cross and the center of the patch have identical horizontal coordinates.

The mushroom EBG is described by the periodicity $D_{E B G}=4.630 \mathrm{~mm}$, the width of patches $P_{E B G}=4.330 \mathrm{~mm}$ and the radius of vias $R_{E B G}=0.600 \mathrm{~mm}$. The mushroom 
EBG surrounds the circularly polarized patch antenna to suppress surface waves on one hand and to form the bottom plane of the FP resonator on the other hand.

The inductive and capacitive (LC) PRS consists of an inductive grid (the bottom surface of the PRS) and a capacitive grid (the top surface of the PRS). The PRS allows us to obtain a phase shift of the reflection coefficient between $+180^{\circ}$ and $-180^{\circ}$. The PRS is designed for the substrate with $h_{1}=1.016 \mathrm{~mm}$ and $\varepsilon_{r 1}=10.2$. The width of strips of the inductive grid is $W_{P R S}=1.310 \mathrm{~mm}$. The width of patches of the capacitive grid is $C_{P R S}=3.190 \mathrm{~mm}$. Both grids are of the same periodicity $D_{P R S}=3.940 \mathrm{~mm}$.

\section{Simulation of EBG and PRS}

The antenna structure specified in the previous section was analyzed in CST Microwave Studio. In Fig. 2, the frequency response of the reflection phase is depicted by the black line, and the frequency response of the magnitude of the surface wave transmission is given by the red line for the mushroom-like electromagnetic bandgap (EBG) structure. Obviously:

- The mushroom-like EBG exhibited a positive reflection phase $\varphi_{1}=136.9^{\circ}$ at $10 \mathrm{GHz}$. Thanks to the positive phase of the reflection coefficient, the distance between the EBG and the partially reflective superstrate (PRS) can be minimized.

- The surface wave transmission is strongly suppressed in the interval from $9.23 \mathrm{GHz}$ to $10.7 \mathrm{GHz}$.

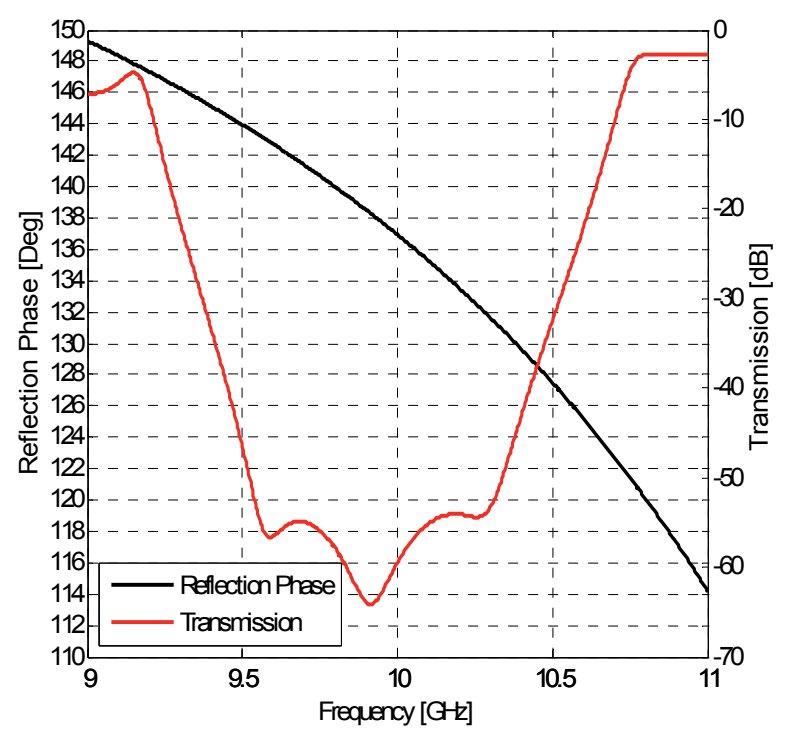

Fig. 2. Frequency response of the reflection phase (black) and surface wave transmission (red) of the mushroom-like EBG structure.

The investigated mushroom EBG consisted of $17 \times 17$ cells.

Figure 3 shows frequency response of the reflection magnitude and the reflection phase of the inductive and capacitive (LC) PRS.
Considering the Fabry-Perot (FP) resonance condition (1), the LC PRS can be used for the design of a high-gain low-profile antenna. We studied two versions of the FP resonator antenna in CST:

- The height of the resonant cavity was $h_{C}=1.500 \mathrm{~mm}$ ( $\lambda / 20.0$; black lines in Fig. 3). At $10 \mathrm{GHz}$, the superstrate exhibited the reflection magnitude 0.94 and reflection phase $\varphi_{2}=-94.4^{\circ}$. According to (2), the maximal obtained gain was $G=15.0 \mathrm{dBi}$.

- The height of the cavity was $h_{C}=0.100 \mathrm{~mm}(\lambda / 300$; red lines in Fig. 3). At $10 \mathrm{GHz}$, the LC PRS showed the reflection magnitude 0.98 and the reflection phase $\varphi_{2}=-134.6^{\circ}$. According to (2), we were able to reach the maximal gain $G=20.0 \mathrm{dBi}$.

In both the cases, the LC PRS consisted of $21 \times 21$ cells.

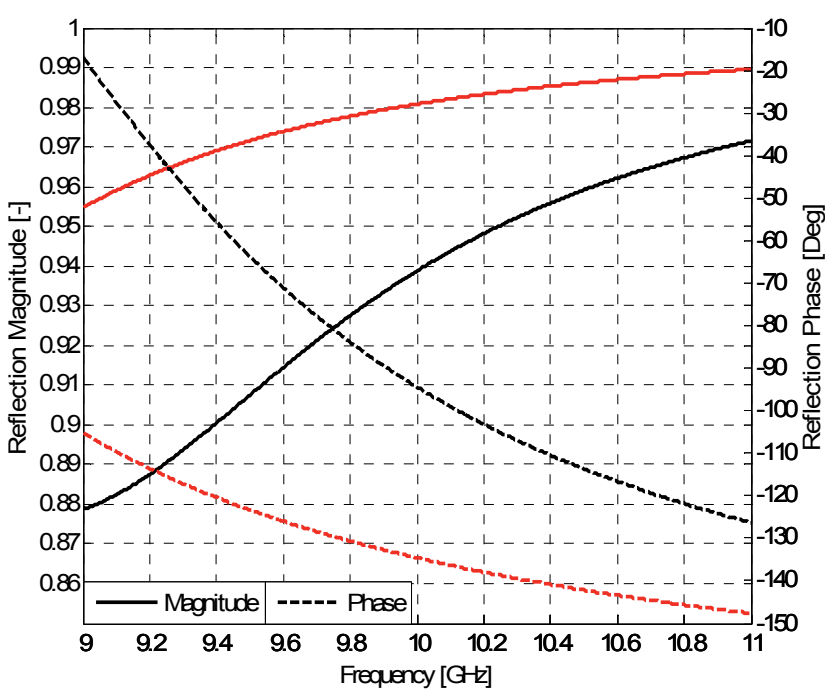

Fig. 3. Frequency response of the reflection magnitude and the reflection phase of the LC PRS for the height of the cavity $h=\lambda / 22$ (black) and $h=\lambda / 300$ (red).

Due to fabrication aspects, we have chosen the resonant cavity of the height $\lambda / 20.0$. Then, the resonator was optimized, and the height of the cavity was reduced from $h_{\mathrm{C}}=1.500 \mathrm{~mm}$ to $h_{C, \text { opt }}=1.400 \mathrm{~mm}(\lambda / 21.4)$.

The optimization also changed the width of strips $W_{P R S}$ from $1.310 \mathrm{~mm}$ to $1.330 \mathrm{~mm}$ (inductive cells), and the width of patches $C_{P R S}$ from $3.190 \mathrm{~mm}$ to $3.430 \mathrm{~mm}$ (capacitive cells). The periodicity stayed unchanged $D_{P R S}=3.940 \mathrm{~mm}$.

Numerical analyses and optimizations of EBG and PRS were based on unit-cell simulations. Simulations were performed in CST Microwave Studio following the procedure described in [17].

\section{Experimental Verification}

Using a conventional printed circuit board (PCB) technology, the simulated antenna was manufactured from commercially available microwave substrates ARLON 

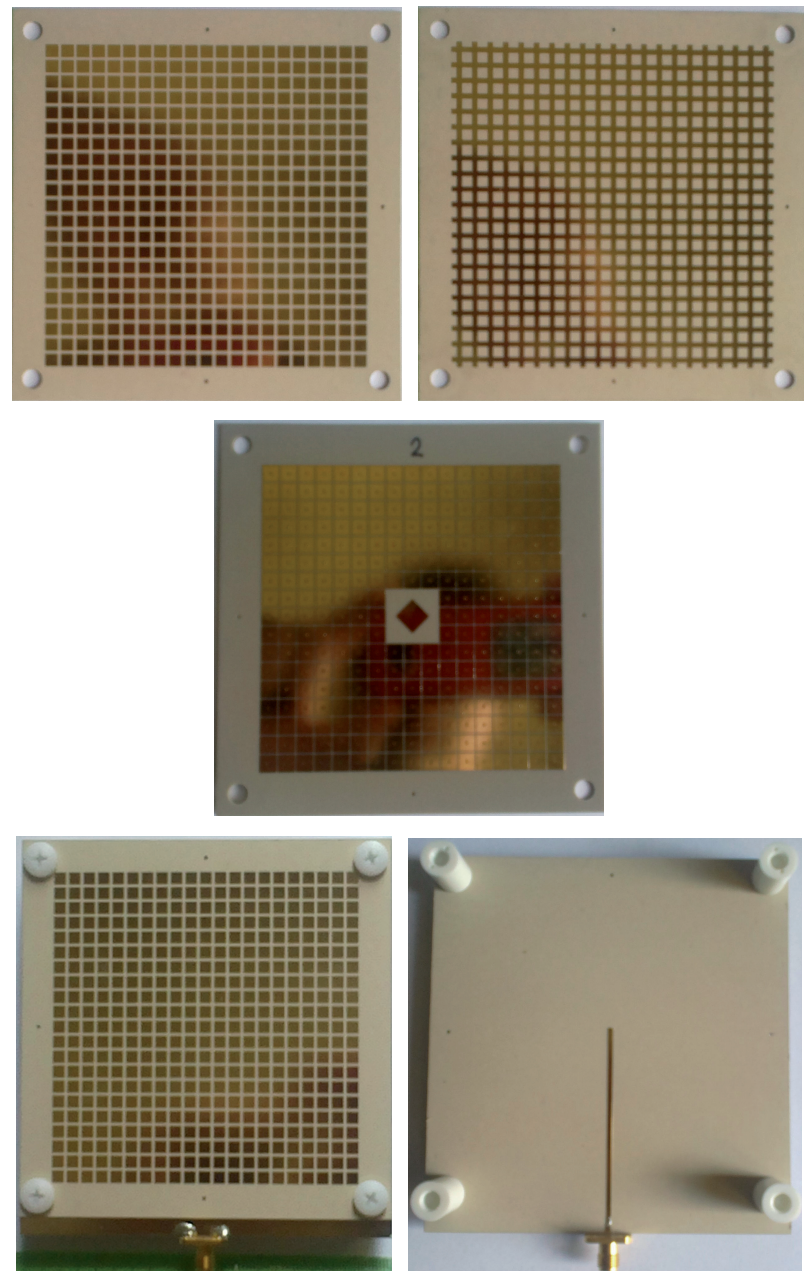

Fig. 4. Photograph of the manufactured antenna: PRS top view (top left). PRS bottom view (top right), patch and EBG (center), complete antenna top view (bottom left), and complete antenna bottom view (bottom right).

$\operatorname{AD} 1000\left(\varepsilon_{r}=10.2, \tan \delta=0.0023\right)$ and ARLON $25 \mathrm{~N}$ $\left(\varepsilon_{r}=3.38, \tan \delta=0.0025\right)$. Photograph of the manufactured prototype of the antenna is shown in Fig. 4. In order to validate simulations experimentally, we measured frequency response of the magnitude of reflection coefficient and axial ratio at the input port of the antenna (Fig. 5 and 6) and radiation patterns at $10 \mathrm{GHz}$ (Fig. 7). For measure-

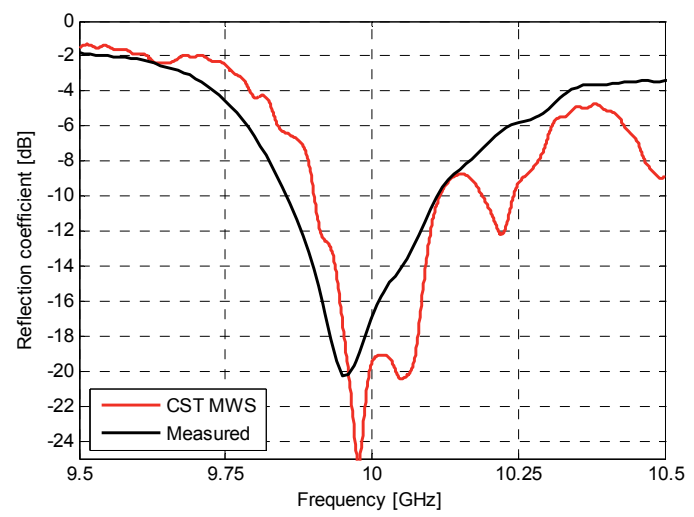

Fig. 5. Simulated (red) and measured (black) frequency response of the magnitude of reflection coefficient at the input port of the designed antenna.

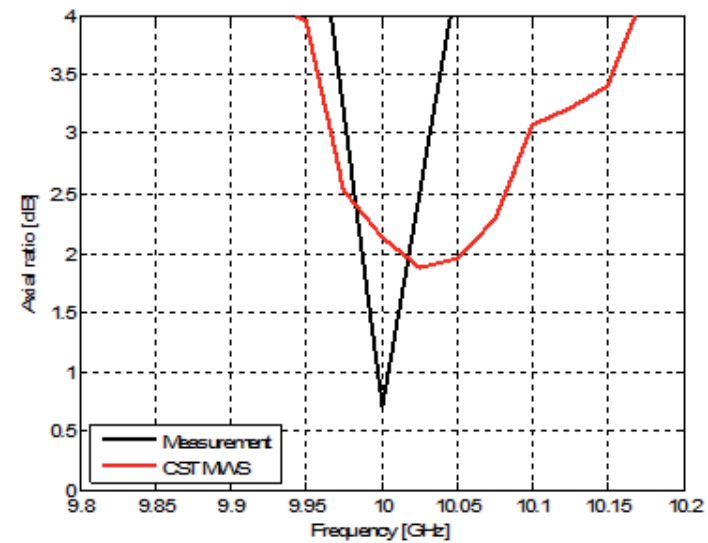

Fig. 6. Simulated (red) and measured (black) frequency response of the axial ratio of the designed antenna.
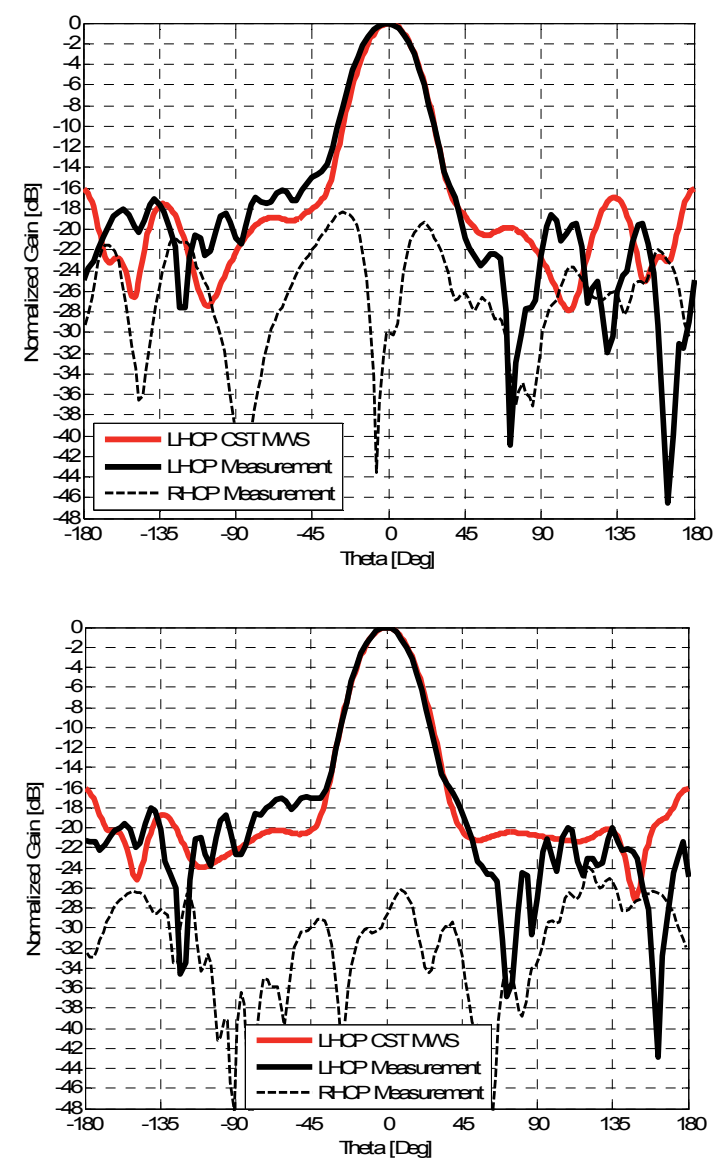

Fig. 7. Simulated (red) and measured (black) normalized radiation patterns of the complete antenna structure at $10 \mathrm{GHz}$ : xz-plane (top), yz-plane (bottom).

ments, we used the vector network analyzer Rohde \& Schwarz ZVA67. Patterns and axial ratio were measured in an anechoic chamber in the far field. In Fig. 5, computed and measured frequency responses of the magnitude of reflection coefficient at the input port of the whole antenna are compared. The measured impedance bandwidth is $2.3 \%$ for $\left|S_{11}\right|<-10 \mathrm{~dB}$.

The measured axial ratio (Fig. 6) shows the bandwidth $0.6 \%$ (versus $1.4 \%$ computed in CST Microwave Studio) for $A R<3.0 \mathrm{~dB}$. The axial ratio is strongly influ- 


\begin{tabular}{|l|c|c|}
\hline & SLL, xz plane & SLL, yz plane \\
\hline Single Antenna & $-10.6 \mathrm{~dB}$ & $-9.6 \mathrm{~dB}$ \\
\hline Antenna + EBG & $-13.6 \mathrm{~dB}$ & $-14.2 \mathrm{~dB}$ \\
\hline Antenna + EBG + PRS & $-21.7 \mathrm{~dB}$ & $-20.8 \mathrm{~dB}$ \\
\hline
\end{tabular}

Tab. 1. Influence of the EBG and PRS on the side lobe level (SLL) of the designed antenna; computed in CST Microwave Studio at $10 \mathrm{GHz}$.

enced by the composition of periodic layers. The antenna generates left-hand circular polarization.

Figure 7 shows the simulated radiation patterns and measured ones at $10 \mathrm{GHz}$. The mushroom-like EBG structure plays an important role in suppressing the level of side lobes due to the band-gap. Hence, the radiation pattern is smoother with reduced backward radiation (see Tab. 1). The gain of the complete antenna structure computed in CST Microwave Studio was $G=15.0 \mathrm{dBi}$.

Finally, we can summarize:

- Simulations and measurements are in a good agreement.

- The profile of the designed antenna was significantly reduced compared to existing antenna concepts $(\lambda / 21$ versus to $\lambda / 2$ or $\lambda / 3$ ).

- The gain of the designed antenna is comparable to the gain of existing antenna concepts $(15 \mathrm{dBi}$ versus 15 to $17 \mathrm{dBi})$.

- The impedance bandwidth of the designed antenna is $2.3 \%$ for $\left|S_{11}\right|<-10 \mathrm{~dB}$.

- The antenna radiates circularly polarized waves. The axial ratio bandwidth is $0.6 \%$ for $A R<3.0 \mathrm{~dB}$.

- Simulations show that radiation efficiency of the antenna is $92.7 \%$, and the total efficiency is equal to $89.6 \%$.

Since the described microwave antenna shows promising parameters, we are going to apply the verified concept to the design of a source of circularly polarized terahertz waves. Since simulations and measurements are in a good agreement, parameters of the terahertz source are verified by simulations only.

\section{Towards Terahertz Frequencies}

In this paragraph, we study a potential conversion of the designed circularly polarized FP antenna to a source of terahertz waves. Since low efficiency and linear polarization are major problems of terahertz antennas, higher efficiency and circular polarization are the main ambitions of this conversion.

Research of photoconductive antennas has started in 1980s. Since then, many photoconductive antennas have been developed. The principle of operation of photoconductive antennas is quite different from conventional microwave antennas. Comparisons of major differences between photoconductive and microwave antennas are given in Tab. 2 [15].

\begin{tabular}{|l|c|c|}
\hline \multicolumn{1}{|c|}{ Parameters } & $\begin{array}{c}\text { Microwave } \\
\text { antennas }\end{array}$ & $\begin{array}{c}\text { Photoconductive } \\
\text { antennas }\end{array}$ \\
\hline Feed-line / source & Transmission line & Laser beam \\
\hline Substrate & $\begin{array}{c}\text { Dielectric material } \\
\text { with thickness }<<\lambda\end{array}$ & $\begin{array}{c}\text { Material thickness } \\
\text { comparable to } \lambda\end{array}$ \\
\hline $\begin{array}{l}\text { Bias } \\
\text { voltage }\end{array}$ & No & Yes \\
\hline $\begin{array}{l}\text { Impedance } \\
\text { matching }\end{array}$ & Easy to achieve & Hard to achieve \\
\hline $\begin{array}{l}\text { Manufacturing } \\
\text { process }\end{array}$ & Easy to make & Hard to make \\
\hline $\begin{array}{l}\text { Computer aided } \\
\text { design }\end{array}$ & Available & Not available \\
\hline
\end{tabular}

Tab. 2. Comparisons of microwave antennas and photoconductive ones.

We can summarize:

- Feed-line/source [15]:

For conventional antennas, various feed lines can be used (microstrip line, coplanar waveguide, etc.). For photoconductive antennas, no feed line exists in reality. Development of low loss terahertz transmission lines is very challenging. Actually, laser plays the role of the feed line for photoconductive antennas.

- Substrate [15]:

As a substrate for conventional antennas, a low-loss dielectric material is used. As a substrate for terahertz antennas, we use photoconductive materials which are basically semiconductors ( $\mathrm{Si}$, InAs, ZnTe, GaSe, InP, SOS, GaAs, and InGaAs). Low-temperature-grown GaAs (LT-GaAs) has become the most popular material because of short photo-carrier lifetime (about $0.25 \mathrm{ps})$, high electric breakdown field $(>5105 \mathrm{~V} / \mathrm{cm})$ and high mobility $\left(>200 \mathrm{~cm}^{2} / \mathrm{Vs}\right)$. The thickness of the substrate of photoconductive antennas has to be comparable with the terahertz wavelength. Otherwise, unwanted substrate modes can be excited.

- Bias voltage [15]:

Whereas conventional antennas do not require a bias voltage, photoconductive antennas when used like transmitting ones require that.

- Impedance matching [15]:

Since no transmission lines are available for photoconductive antenna, no tuning circuit can ensure the impedance matching. The impedance matching is an issue for photoconductive antennas.

- Manufacturing [15]:

Terahertz antennas are usually fabricated by a conventional lithography on low temperature GaAs grown at about $250^{\circ} \mathrm{C}$ and post annealed at a higher temperature (but $<600^{\circ} \mathrm{C}$ ). The metal patterns are deposited using one of the usual schemes for resistive contact on n-type GaAs.

- Computer aided design [15]:

There are no complete design tools available for the design of photoconductive antennas. These antennas are mainly developed by trial-and-error. Only metallic 
structures may be simulated using electromagnetic (EM) simulation tools.

Let turn the attention to the conversion of the developed microwave circularly polarized FP antenna (Fig. 1) to the antenna operating at terahertz frequencies. When redesigning the antenna, the following modifications have to be done:

- All three layers of the substrate are replaced by gallium arsenide $\left(\varepsilon_{r}=12.94, \tan \delta=0.006\right)$. The bottom layer is $400 \mu \mathrm{m}$ high. The middle layer and the top one are $15 \mu \mathrm{m}$ high.

- The microstrip transmission line on the bottom side of the structure is replaced by a photo-mixer which transforms the beat signal of the incident laser wave into terahertz waves using a photoconductor and an appropriate antenna. We can use slot dipoles if a large bandwidth is not required.

The interdigital electrode capacitance of the photomixer can increase the antenna resistance. Moreover, we can change the capacitance to tune the system for the operation at a given frequency.

- For the operation frequency $f=1 \mathrm{THz}$ and the height of the cavity $h=\lambda / 150$, we have to re-compute dimensions of the EBG $\left(D_{E B G}=35.08 \mu \mathrm{m}, P_{E B G}=\right.$ $\left.19.38 \mu \mathrm{m}, R_{E B G}=7.00 \mu \mathrm{m}\right)$, size of the PRS $\left(D_{P R S}=\right.$ $\left.17.97 \mu \mathrm{m}, C_{P R S}=14.64 \mu \mathrm{m}, W_{P R S}=6.30 \mu \mathrm{m}\right)$, dimensions of the cross-slot aperture $\left(L_{S 1}=23.24 \mu \mathrm{m}\right.$, $\left.L_{S 2}=42.45 \mu \mathrm{m}\right)$, and patch size $\left(L_{S 1}=31.98 \mu \mathrm{m}\right)$.

In Fig. 8, axial ratio of the terahertz antenna is depicted. Simulations in CST Microwave Studio show that the axial ratio bandwidth is $1.4 \%$ for $A R<3.0 \mathrm{~dB}$. The final antenna generates left-handed circular polarization. The microwave antenna (Fig. 6) exhibited the computed axial ratio $1.4 \%$ for $A R<3.0 \mathrm{~dB}$ and left-hand circular polarization.

Figure 9 shows simulated radiation patterns of the terahertz antenna at the frequency $1 \mathrm{THz}$. Let us compare simulation results for the terahertz antenna and the microwave one (Fig. 7).

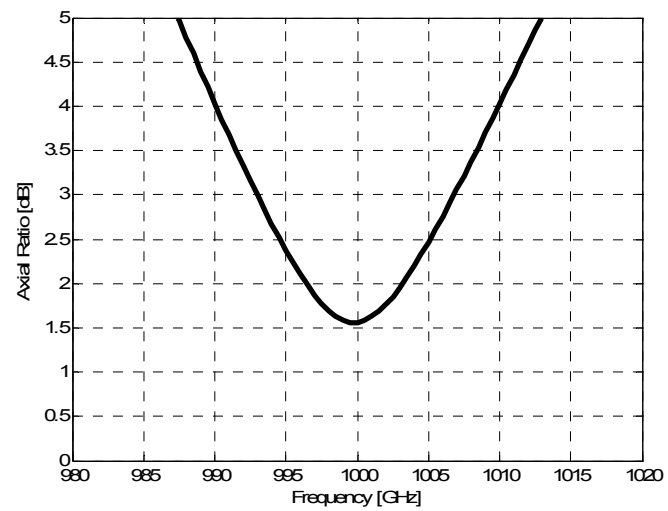

Fig. 8. Simulated frequency response of the axial ratio of the terahertz antenna in broadside direction.
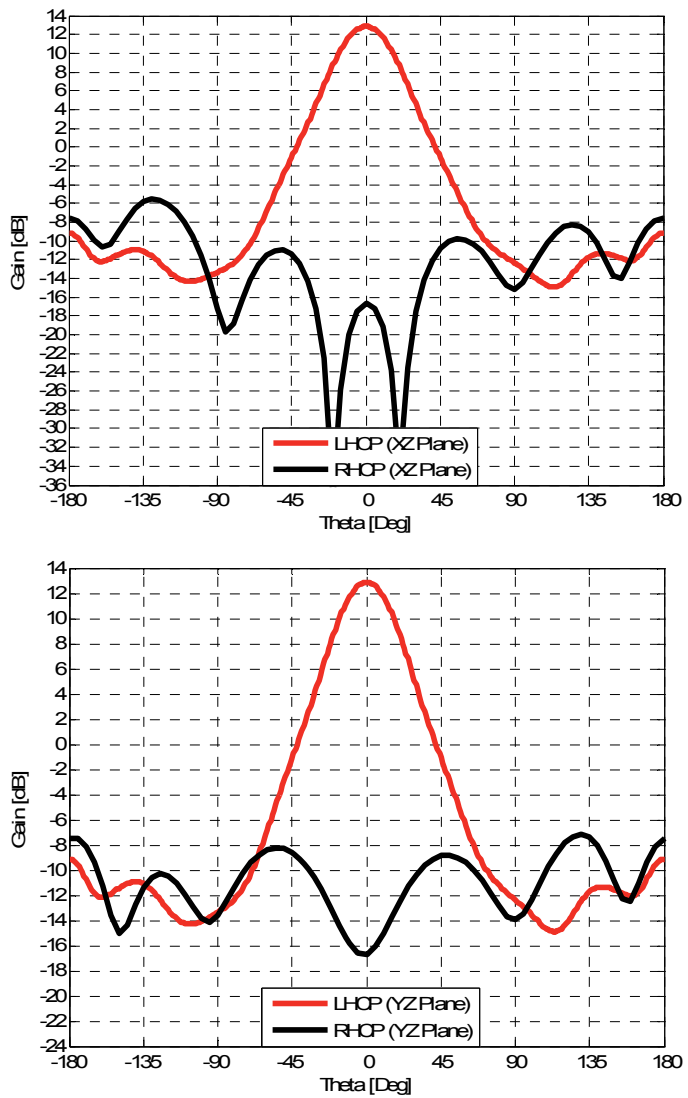

Fig. 9. Simulated radiation patterns of terahertz circularly polarized FP antenna at $1 \mathrm{THz}$ : xz-plane (top), yz-plane (bottom).

For both the antennas:

- The gain about $15 \mathrm{dBi}$ is obtained;

- The side lobe level is about $-20 \mathrm{~dB}$;

- The axial ratio bandwidth is lower than $2 \%$.

Simulations show that radiation efficiency of the terahertz antenna is $43.6 \%$ and total efficiency equals $42.4 \%$ if optical-to-terahertz conversion is not considered.

\section{Summary}

In the paper, a low profile circularly polarized antenna with a superstrate lens was described. The antenna was designed for $f=10 \mathrm{GHz}$. Using a negative-phase LC superstrate and a positive-phase mushroom-like EBG structure surrounding the antenna, a cavity of Fabry-Perot resonator was formed. In numerical studies, the height of the cavity antenna was reduced from $\lambda / 2$ to $\lambda / 20$ and $\lambda / 300$.

The designed antenna exhibits low profile, circular polarization, and increased forward radiation gain. These properties were achieved by an original combination of two approaches:

- A mushroom-like EBG structure was used to surround the patch. That way, surface waves were suppressed and the antenna gain was increased.

- A novel LC superstrate was developed to allow us 
an arbitrary setting of the phase shift of the reflection coefficient. That way, the height of the FP resonator was reduced significantly.

Compared to existing approaches [11-14], the profile of the designed antenna was reduced $(\lambda / 21$ versus $\lambda / 2$ or $\lambda / 3)$. On the other hand, the gain of the designed antenna was similar (about $15 \mathrm{dBi}$ ), and the impedance bandwidth of the designed antenna was comparable (about 2.3\% for $\left.\left|\mathrm{S}_{11}\right|<-10 \mathrm{~dB}\right)$. The antenna radiated circularly polarized waves, and the axial ratio bandwidth was $0.6 \%$ for $A R<$ $3.0 \mathrm{~dB}$.

Obviously, the axial ratio bandwidth of the antenna is narrow, and should be improved for practical applications. The ongoing research is therefore focused on achieving a wider bandwidth of a LC superstrate.

The antenna concept was shown to be applicable at terahertz frequencies. When redesigning the antenna, substrates have to be replaced by gallium arsenide, feeder has to be replaced by photomixer and the dimensions of antenna components have to be related to the terahertz wavelength. Computer simulations show that parameters of the terahertz antenna and the microwave one are similar.

\section{Acknowledgments}

The presented research was financed by the Czech Science Foundation under grant no. P102/12/1274. Research is a part of the COST Action IC 1102 VISTA (grant LD12012 of the Czech Ministry of Education). For simulations and measurements, infrastructure of the SIX Center was used.

\section{References}

[1] BOUTAYEB, H., MAHDJOUBI, K., TAROT, A. C., DENIDNI, T.A. Directivity of an antenna embedded inside a Fabry-Perot cavity: analysis and design. Microwave and Optical Technology Letters, 2006, vol. 48, no. 1, p. 12-17. ISSN: 1098-2760. DOI: $10.1002 /$ mop. 21249

[2] FERESIDIS, A. P., GOUSSETIS, G., WANG, S. H., VARDAXOGLOU, C. J. Artificial magnetic conductor surfaces and their application to low-profile high-gain planar antennas. IEEE Transactions on Antennas and Propagation, 2005, vol. 53, no. 1, p. 209-215. DOI: 10.1109/TAP.2004.840528

[3] SIEVENPIPER, D., ZHANG, L., BROAS, R. F. J., ALEXOPOULOS, N. G., YABLONOVITCH, E. High-impedance electromagnetic surfaces with a forbidden frequency band. IEEE Transactions on Microwave Theory and Techniques, 1999, vol. 47, no. 11, p. 2059-2074. ISSN: 0018-9480. DOI: 10.1109/22.798001

[4] WEILY, A. R., HORVATH, L., ESSELlE, K. P., SANDERS, B. C., BIRD, T. S. A planar resonator antenna based on a woodpile EBG material. IEEE Transactions on Antennas and Propagation, 2005, vol. 53, no. 1, p. 216-223. ISSN: 0018-926X. DOI: 10.1109/TAP.2004.840531

[5] LI, L. L., LEI, S., LIANG, C. H. Metamaterial-based Fabry-Perot resonator for ultra-low profile high-gain antenna. Microwave and Optical Technology Letters, 2012, vol. 54, no. 11, p. 2620-2623. ISSN: 1098-2760. DOI: 10.1002/mop.27155

[6] LI, Y, ESSELLE, K. P. Small EBG resonator high-gain antenna using in-phase highly-reflecting surface. Electronics Letters, 2009 , vol. 45, no. 21 , p 1058-1060. ISSN: 0013-5194. DOI: $10.1049 / \mathrm{el} .2009 .0959$

[7] ZHOU, L., LI, H. Q., QIN, Y. Q., WEI, Z. Y., CHAN, C. T. Directive emissions from sub wavelength metamaterial-based cavities. Applied Physics Letters, 2005, vol. 86, no. 10, p. 101101. ISSN: 0003-6951. DOI: 10.1063/1.1881797

[8] OURIR, A., DE LUSTRAC, A., LOURTIOZ, J. M. Optimization of metamaterial based sub wavelength cavities for ultra-compact directive antennas. Microwave and Optical Technology Letters, 2006 , vol. 48 , no. 12 , p. $2573-2577$. ISSN: 1098-2760. DOI: 10.1002/mop.21996

[9] PITRA, K., RAIDA, Z., HARTNAGEL, H. L. Design of circularly polarized terahertz antenna with interdigital electrode photomixer. In Proceedings of the 7th European Conference on Antennas and Propagation EuCAP 2013. Gothenburg (Sweden), 2013, p. 24312434. ISBN: 978-88-907018-3-2

[10] GARG, R., BHARTIA, P., BAHL, I., ITTIPIBOOM, A. Microstrip Antenna Design Handbook. Norwood (USA): Artech House, 2001. ISBN: 0-89006-513-6

[11] ZEB, B. A., ESSELLE, K. P. A partially reflecting surface with polarization conversion for circularly polarized antennas with high directivity. In Proceedings of the International Conference on Electromagnetics in Advanced Applications ICEAA 2012. Cape Town (South Africa), 2012, p. 466-469. ISBN: 978-1-4673-03330, DOI: 10.1109/ICEAA.2012.6328672

[12] WANGWANG, H., JUN, O., GUO, Z., LI, Y., YANG, F. A single-feed high-gain Fabry-Perot antenna with reconfigurable polarization capability. In Proceedings of the Conference on Cross Strait Quad-Regional Radio Science and Wireless Technology CSQRWC 2013. Chengdu (China), 2013, p. 279-281. DOI: 10.1109/CSQRWC.2013.6657408

[13] BO ZHU, ZHI NING CHEN, YIJUN FENG Fully substrate-integrated high-gain thin Fabry-Perot cavity antennas. In Proceedings of Asia-Pacific Microwave Conference APMC 2011, Melbourne (Australia), 2011, p. 602-605. ISBN: 978-1-4577-2034-5

[14] VAIDYA, A. R., GUPTA, R. K., MISHRA, S. K., MUKHERJEE, J. Right-hand/left-hand circularly polarized high-gain antennas using partially reflective surfaces. IEEE Antennas and Wireless Propagation Letters, 2014, vol. 13, p. 431-434. ISSN: 1536-1225. DOI: 10.1109/LAWP.2014.2308926

[15] HUANG, Y., KHIABANI, N., SHEN. Y., LI, D. Terahertz photoconductive antenna efficiency. In Proceedings of the International Workshop on Antenna Technology iWAT 2011. Hong Kong (China), 2011, p. 152-156. ISBN: 978-1-4244-9133-9. DOI: 10.1109/IWAT.2011.5752384

[16] HASHMI, R. M., ZEB, B. A., ESSELLE, K.P. Wideband highgain EBG resonator antennas with small footprints and all-dielectric superstructures. IEEE Transactions on Antennas and Propagation, 2014, vol. 62, no. 6, p. 2970-2977. ISSN: 0018-926X. DOI: 10.1109 /TAP.2014.2314534

[17] KOVÁCS, P., RAIDA, Z. Design and optimization of high-impedance surfaces. International Journal of $R F$ and Microwave Computer-Aided Engineering, 2012, vol. 22, no. 4, p. 541-544. ISSN: 1096-4290. DOI: $10.1002 /$ mmce. 20643

\section{About the Authors ...}

Kamil PÍTRA was born in Strakonice, the Czech Repub- 
lic, in 1984. He received his master degree in 2010 and Ph.D. degree in 2014, both in Electrical Engineering, from the Brno University of Technology. His research interest is focused on antenna design and wideband antenna applications.

Zbynek RAIDA has graduated at Brno University of Technology, Faculty of Electrical Engineering and Communication (FEEC BUT). Since 1993, he has been with the Department of Radio Electronics FEEC BUT, now as a Professor. In 1996 and 1997, he was with the Laboratoire de Hyperfrequences, Universite Catholique de Louvain, Belgium, working on variational methods of numerical analysis of electromagnetic structures. In 2006-2013, he was the head of the Dept. of Radio Electronics, and since 2010 he has been the head of the SIX Center. Together with his students and colleagues, Zbyněk Raida has been working on numerical modeling and optimization of electromagnetic structures, exploitation of artificial neural networks for solving electromagnetic compatibility issues, and the design of special antennas. Zbyněk Raida is a member of IEEE Microwave Theory and Techniques Society.

Jaroslav LÁČÍK received the M.Sc. and Ph.D. degrees from Brno University of Technology, Brno, Czech Republic, in 2002 and 2007, respectively. He is currently an Associate Professor at the Department of Radio Electronics, Faculty of Electrical Engineering and Communication, Brno University of Technology. His research interests are antennas, body-centric wireless communication, and computational electromagnetics. He is a member of IEEE.

\section{Feature Articles}

In 2015, the Radioengineering Journal started to publish feature articles describing latest development in selected areas of wireless communication. Since feature articles have been accepted by readers well, we are going to continue their publication in 2016. Here is the tentative list:

\section{April 2016}

\section{- Source concept for optimal antennas}

Pavel HAZDRA,

Czech Technical University in Prague,

Czech Republic

- Fully photonic wireless link for transmission of synchronization signals

Otakar WILFERT and Peter BARCÍK, Brno University of Technology,

Czech Republic

\section{June 2016}

\section{- Chipless RFID}

Milan POLIVKA and Jan MACHAC, Czech Technical University in Prague, Czech Republic

- Antenna solutions fabricated with 3D printing technologies

Cyril LUXEY,

University of Nice-Sophia Antipolis, France

\section{September 2016}

- Advanced multicarrier modulation schemes

Zsolt KOLLAR and Peter HORVATH,

Budapest University of Technology and Economics, Hungary

- Negative impedance converters and small antennas

Daniel SEGOVIA-VARGAS, Carlos III University of Madrid, Spain

\section{December 2016}

- An efficient framework for analysis of wire-grid shielding structures over a broad frequency range

Andrzej KARWOWSKI, Artur NOGA and Tomasz TOPA,

Silesian University of Technology, Gliwice, Poland

- Modeling tree attenuation and scattering

Fernando PEREZ-FONTAN,

University of Vigo,

Spain

Milan KVICERA and Pavel PECHAC, Czech Technical University in Prague, Czech Republic

Jonathan ISRAEL, ONERA, France 